\title{
CD8T-cell-mediated protection against liver-stage malaria: lessons from a mouse model
}

\section{Natalija Van Braeckel-Budimir and John T. Harty*}

Department of Microbiology, University of lowa, lowa, IA, USA

\section{Edited by:}

Ute Frevert, New York University School of Medicine, USA

Reviewed by:

Hridayesh Prakash, University of Hyderabad, India

Jieliang Li, Temple University, USA

*Correspondence:

John T. Harty, Department of

Microbiology, University of lowa,

3-501 Bowen Science Building, 51

Newton Road, lowa City,

IA 52242-1109, USA

e-mail: john-harty@uiowa.edu

\begin{abstract}
Malaria is a major global health problem, with severe mortality in children living in sub-Saharan Africa, and there is currently no licensed, effective vaccine. However, vaccineinduced protection from Plasmodium infection, the causative agent of malaria, was established for humans in small clinical trials and for rodents in the 1960s. Soon after, a critical role for memory CD8 T cells in vaccine-induced protection against Plasmodium liver-stage infection was established in rodent models and is assumed to apply to humans. However, these seminal early studies have led to only modest advances over the ensuing years in our understanding the basic features of memory CD8 T cells required for protection against liver-stage Plasmodium infection, an issue which has likely impeded the development of effective vaccines for humans. Given the ethical and practical limitations in gaining mechanistic insight from human vaccine and challenge studies, animal models still have an important role in dissecting the basic parameters underlying memory CD8 T-cell immunity to Plasmodium. Here, we will highlight recent data from our own work in the mouse model of Plasmodium infection that identify quantitative and qualitative features of protective memory CD8 T-cell responses. Finally, these lessons will be discussed in the context of recent findings from clinical trials of vaccine-induced protection in controlled human challenge models.
\end{abstract}

\section{Keywords: CD8 T cells, memory, protection, Plasmodium, mice, humans}

\section{INTRODUCTION}

Malaria represents an enormous global health problem. It is associated with around 200 million reported annual cases and more than 600,000 deaths, most of them recorded in sub-Saharan Africa (WHO, 2011). Current disease treatment is limited to antimalarial drugs targeting the symptomatic blood-stage infection. Given the enormous genetic plasticity of the parasite, the emergence of antimalarial drug resistance is inevitable and thus a major concern (Miller et al., 2013). Hence, the development of a protective malaria vaccine is alarmingly urgent.

Blocking Plasmodium infection at the level of the silent, liver stage of malaria represents an attractive strategy for disease prevention (Kappe et al., 2010). The earliest evidence of vaccineinduced, sterile, liver-stage immunity originates from mouse studies, in which it was demonstrated that vaccination with radiation attenuated sporozoites (RAS) prevented development of blood-stage Plasmodium berghei infection after sporozoite challenge (Nussenzweig et al., 1967, 1969). Importantly, RAS-induced sterile protection was confirmed in human subjects, in whom it was induced upon exposure to bites of more than 1000 sporozoitebearing, irradiated mosquitoes (Clyde et al., 1973; Edelman et al., 1993; Hoffman et al., 2002). Persistent efforts to repeat the success of RAS in inducing sterilizing immunity using different vaccine formulations and regimens (e.g., which are easier to manufacture and can be administered through approved vaccination routes) have led only to a partial success (Moorthy et al., 2004; Dunachie et al., 2006; Bejon et al., 2007; Moorthy and Ballou, 2009; Agnandji et al., 2012). Therefore, RAS immunization still represents the gold standard for induction of sterile protection and, despite logistical challenges, has moved recently to Phase I clinical trial.

Besides being a potential vaccine candidate, RAS has been used as an invaluable tool for studying protective immune responses against liver-stage Plasmodium infections. Although the earliest work describes neutralizing antibodies as the main mechanism of RAS-induced protection (Potocnjak et al., 1980; Yoshida et al., 1980), more recently depletion studies and adoptive-transfer experiments have demonstrated that CD8 T cells have a leading role in protection against sporozoite challenge (Schofield et al., 1987; Weiss et al., 1988; Rodrigues et al., 1991). The critical role of CD8 T cells in protection against liver-stage malaria has been confirmed upon immunization with different formulations, such as recombinant vaccines [e.g., Salmonella typhimurium, vaccinia virus or adeno virus expressing $P$. berghei circumsporozoite (CS) protein] and genetically attenuated parasite (GAP; Sadoff et al., 1988; Lanar et al., 1996; Rodrigues et al., 1997; Jobe et al., 2007).

Despite the strong evidence for the role of CD8 T cells in sterile protection against malaria, critical qualitative and quantitative characteristics of the protective response and effector mechanisms engaged by CD8 T cells remain incompletely understood. The modest progress in this field is strongly influenced by the extreme genetic plasticity of the parasite, its complex life cycle and the paucity of defined antigenic targets for CD8 T cells. Filling these knowledge gaps is of an utmost importance, as this information would facilitate the development of successful pre-erythrocytic vaccine candidates. 
Although highly desirable, studies of Plasmodium infection in humans are limited by multiple ethical and practical factors (inability to manipulate the immune response for mechanistic studies, difficulty to access relevant samples, etc.). Therefore, progress in understanding immunity against liver-stage malaria critically depends on the availability of suitable animal models. Infection of mice with $P$. berghei and Plasmodium yoelii, two rodent Plasmodium pathogens, closely resembles the early stages of human liver invasion, replication, and development within hepatocytes (Meis et al., 1983; Sturm et al., 2006; Baer et al., 2007). Additionally, clear differences in infectivity and pathogenicity displayed by these two Plasmodium species mimic the diversity of human Plasmodium infections (Sedegah et al., 2007). Therefore, the mouse model has proven to be invaluable for basic studies, such as host-parasite interactions and the underpinning of the immune mechanisms driving protection induced upon vaccination and sporozoite inoculation.

Here, we will present and highlight lessons about protective memory CD8 T-cell thresholds for liver-stage protection and effector mechanisms engaged by these cells, learned from vaccinations of mice with both subunit (Plasmodium CS-derived epitope) and whole-parasite (RAS and GAP) vaccine formulations. Finally, we will discuss the outcomes of recent vaccine clinical trials in light of our own findings and highlight the implications of the lessons learned for further development of liver-stage malaria vaccines.

\section{INDUCTION OF CD8 T CELLS BY SUBUNIT VACCINES SETTING THE NUMERIC THRESHOLD}

Induction of sterilizing immunity against liver-stage parasite represents a challenge for at least two reasons. First, liver-residing or recruited CD8 T cells have to locate and eliminate all the infected hepatocytes/parasites to prevent progression of the infection from the liver to the blood stage. A single mosquito bite delivers a few hundred infectious sporozoites into the skin dermis. Given that only a fraction of sporozoites actually reaches the liver and infects hepatocytes, we estimate that only 1 out of $10^{9}$ hepatocytes in humans or 1 out of $10^{6}$ hepatocytes in mice are infected after mosquito infection. Similarly, low frequencies of infected hepatocytes are likely after intravenous (i.v.) challenge with 100-1000 virulent sporozoites, as used in many mouse studies. Thus, surveying CD8 T cells target extremely rare events, creating the proverbial "needle in a haystack" scenario. Second, the time interval between the start of the liver-stage infection and release of blood-stage merozoites is very short ( 7 days in Plasmodium falciparum and 2 days in P. berghei/P. yoelii), which means that CD8 T cells have a limited amount of time to perform their task (Sturm et al., 2006; Sturm and Heussler, 2007; Todryk and Hill, 2007). In the context of these spatial and temporal pressures, it is important to gain knowledge about the quantitative and qualitative features of protective memory CD8 T-cell response.

To address these questions, we used a mouse model to induce a stable, long-lasting memory CD8 T-cell response against a defined epitope ( $P$. berghei $\mathrm{CS}_{252-260}$ ). For this purpose, we exploited an accelerated prime-boost approach that is well established in our laboratory (Badovinac et al., 2005). In short, mice were vaccinated with mature dendritic cells (DCs), which had been incubated for
$2 \mathrm{~h}$ with CS-derived peptide to allow surface peptide-MHC complexes to form (DC-CS), and a week later they received a booster vaccination with an attenuated Listeria monocytogenes expressing the same CS-derived peptide (LM-CS; Schmidt et al., 2008). This vaccination strategy (from here on abbreviated as DC-LM) has proven to be a robust tool for the generation and study of stable, long-lasting memory CD8 T-cell response against defined antigenic determinants without contribution by other components of the immune response (CD4 T cells, antibodies, NK cells; Schofield et al., 1987; Weiss et al., 1993; Doolan and Hoffman, 1999, 2000).

This vaccination approach allowed us to induce CS-specific CD8 T-cell immune responses with a magnitude of $1-7 \%$ of the total peripheral blood leukocytes (PBLs) (up to 20\% of CD8 T cells). Strikingly, this response was stable and protective against repeated sporozoite challenges for at least 19 months (the life span of a laboratory mouse). Moreover, by titrating the booster vaccine dose, we were able to induce CD8 T-cell response with decreasing magnitude, which allowed us to determine the potential numeric threshold required for protection. Strikingly, we observed that more than 95\% of animals with CD8+ T-cell frequencies exceeding a threshold of $1 \%$ of total PBLs were protected against sporozoite challenge, while more than $95 \%$ of animals with CD8 T-cell frequencies below this threshold developed bloodstage infection and were thus not sterilely protected (Schmidt et al., 2008). These findings demonstrate that sterile protection against sporozoite infection requires a remarkably strong CD8 T-cell response, representing a substantial fraction of the total CD8 T-cell pool and highly exceeding frequencies of antigen-specific CD8 T cells required for plausible protection against various viral and bacterial infections (Schmidt et al., 2008). On the other hand, given the previously mentioned spatial and temporal pressures on the sterilizing CD8 T-cell-mediated response, these results do not come as a complete surprise. It is not difficult to imagine that recognition and elimination of all the rare infected hepatocytes within 2 days require mobilization of extremely high CD8 T-cell numbers.

Thus, we describe a quantitative feature of protective memory CD8 T-cell response against liver-stage Plasmodium, and show that if met, this feature can potentially ensure life-long protection.

\section{DISSECTING EFFECTOR FUNCTIONS UTILIZED BY PROTECTIVE MEMORY CD8 T CELLS}

Various effector molecules, such as IFN- $\gamma$, TNF- $\alpha$, perforin, FasL, and TRAIL, are utilized by memory CD8 T cells in protection against different infections (Raeder et al., 2000; Trapani and Smyth, 2002; Shrestha etal., 2006, 2008; Ishikawa et al., 2009). A few attempts were made to define the effector component of the CD8 T-cell responses against liver-stage Plasmodium infections. As these studies were based on RAS immunizations, which in addition to CD8 T-cell responses also induce also CD4 $\mathrm{T}$ cell and antibody responses, it is still not completely clear which pathways are engaged by memory CD8 T cells (Ferreira et al., 1986; Schofield et al., 1987; Tsuji et al., 1995; Renggli et al., 1997; Rodrigues et al., 2000). Furthermore, it is not clear whether CD8 T-cell responses against different Plasmodium species require the same effector pathways for sterile immunity. This information is of high relevance for the development of human 
vaccines, which would ideally protect against multiple Plasmodium species.

To study effector functions in well-defined memory CD8 T-cell population, we used the DC-LM prime/boost approach. In contrast to vaccination with whole-parasite formulations, which in addition to CD8 $\mathrm{T}$ cells elicit also non-CD8 T-cell responses, this immunization approach allows focus only on effector pathways utilized by memory CD8 T cells (Schmidt et al., 2009). Memory CD8 T-cell responses against $P$. beghei and $P$. yoelii CS-derived peptides were induced in wild-type (wt) BALB/c mice together with mice deficient for various effector molecules (IFN- $\gamma$, perforin, FasL, and TRAIL). Additionally, TNF- $\alpha$ was depleted by neutralizing antibodies in vaccinated, wt mice to assess its role in CD8 T-cell-mediated sterile protection.

The most important finding of the study was that the pathways of memory CD8 T-cell-mediated protection against liver-stage infection were not completely overlapping for the two different Plasmodium species (Butler et al., 2010). Protection against P. berghe $i$ was diminished in the absence of IFN- $\gamma$ and TNF- $\alpha$ but was not influenced by the absence of perforin. In contrast, the absence of perforin, but not TRAIL and FasL, completely eliminated protection against $P$. yoelii. In line with this finding, induction of generalized inflammation by treatment of animals with TLR9 agonist (CpG) 24 h-post sporozoite infection was sufficient to block the progression of $P$. berghei, but not $P$. yoelii infection to the blood stage. Susceptibility of $P$. yoelii sporozoites to $\mathrm{CpG}$-induced inflammation was observed only during a very short window of $12 \mathrm{~h}$-post infection. As $P$. yoelii displays higher infectivity in rodents compared to $P$. berghei (Sedegah et al., 2007), it is likely that more stringent control of parasite replication and development, involving direct killing of infected hepatocytes through the perforin pathway, are required for successful control of this infection at the liver stage.

Thus, effector mechanisms exploited by memory CD8 T cells in protection against liver-stage infection are Plasmodium species specific, a finding of high relevance for development of protective human vaccine targeting clinically relevant $P$. falciparum and $P$. vivax.

\section{INFLUENCE OF Plasmodium-HOST INTERACTIONS ON MEMORY CD8 T-CELL-MEDIATED PROTECTION AGAINST LIVER-STAGE Plasmodium INFECTION}

Although we demonstrated a clear threshold ( $>1 \%$ of PBL) for CD8 T-cell-mediated sterilizing immunity, our findings were limited to one mouse strain $(\mathrm{BALB} / \mathrm{c})$ and one Plasmodium species (P. bergei; Schmidt et al., 2008). The observation that some mouse strains, e.g., C57Bl/6 and B10.D2, are more difficult to protect against sporozoite infection upon RAS vaccination than $B A L B / c$ (Weiss et al., 1989; Doolan and Hoffman, 2000) made us wonder to what extent the protective threshold is influenced by hostparasite interactions defined by host-strain-specific background genes. Multiple studies have acknowledged the important role of non-MHC-linked host background genes on development of an immune response against infections (Hsieh et al., 1995; Diosi, 2002). Of equal importance, human malaria is caused by infection with multiple Plasmodium species, thus raising the question to what extent the protective threshold is influenced by different parasite species.

In our hands, following the DC-LM prime/boost with $P$. yoelii $\mathrm{CS}_{280-288}$, mice developed high-magnitude memory CD8 T-cell responses that exceeded the defined threshold established with P. berghei model ( $>1 \%$ of total $\mathrm{PBL}$ ). Interestingly, unlike our observations after $P$. berghei infection, these animals were highly susceptible even to a low-dose, P. yoelii challenge (Schmidt et al., 2011). Although we were able to substantially increase the size of memory CD8 T-cell population ( $>1.5 \%$ of total PBL), we were unable to find a defined, numerical threshold required for stable (defined as $>80 \%$ ) protection against liver-stage infection with $P$. yoelii. This observation was in line with the finding that protection against $P$. yoelii infection requires more stringent control of parasite replication, achieved by direct killing of infected hepatocytes by engagement of the perforin pathway (Butler et al., 2010). Thus, the protective memory CD8 T-cell threshold was highly influenced by the species of Plasmodium even in a single mouse strain.

In parallel with this, we observed that different strains of mice expressing the same MHC class I molecule $\left(\mathrm{H}-2 \mathrm{~K}^{\mathrm{d}}\right)$ that presents the CS-epitopes, but differing in background genes, display dramatically different levels of susceptibility to $P$. berghei sporozoite challenge (Figure 1). Importantly, in all these mouse strains, the DC-CS/LM-CS vaccination induced memory CD8 T-cell responses of similar magnitude and quality (e.g., production of IFN- $\gamma$; Schmidt et al., 2011). While more than $80 \%$ of immunized $\mathrm{BALB} / \mathrm{c}$ and $\mathrm{DBA} / 2$ mice were protected against the infection, protection observed in mice with $\mathrm{C} 57 \mathrm{Bl} / 6$ or closely related $\mathrm{C} 57 \mathrm{Bl} / 10$ background genes (CB6F1 and B10.D2, respectively) was marginal or completely absent. Only after administration of a second booster vaccination dose, which induced extremely high-magnitude memory CD8 T-cell response ( $\sim 15 \%$ of the total PBL or $\sim 60 \%$ of the total CD $8+\mathrm{T}$ cells), were B10.D2 and CB6F1 mice protected from sporozoite challenge. Furthermore, a study performed on reciprocal bone marrow chimeras between B10.D2 and BALB/c mice revealed that B10.D2 T-cell immune response reconstituted on a BALB/C background displays a superior protection in comparison to $\mathrm{BALB} / \mathrm{c}$ T-cell response reconstituted on $\mathrm{C} 57 \mathrm{Bl} / 10$ background. All the evidence results suggest that it is not the functional or quantitative property of memory CD8 T-cell response, but rather the host-pathogen interactions, determined by the hostbackground gene milieu and parasite species, that are key factors determining thresholds for memory CD8 T-cell-mediated protection. Of note, we have not identified a mouse background that is easier to protect against Plasmodium sporozoite infection.

This knowledge obtained in a mouse malaria model is of a particular relevance for understanding differences in protection observed in heterogeneous human populations and for development of successful malaria vaccines, which have to ensure protection against multiple, human Plasmodium species. Given the modest success of current human vaccines in inducing CD8 T-cell response, the extremely high numerical requirements for sterile protection against malaria may be an additional challenge for vaccine development. 


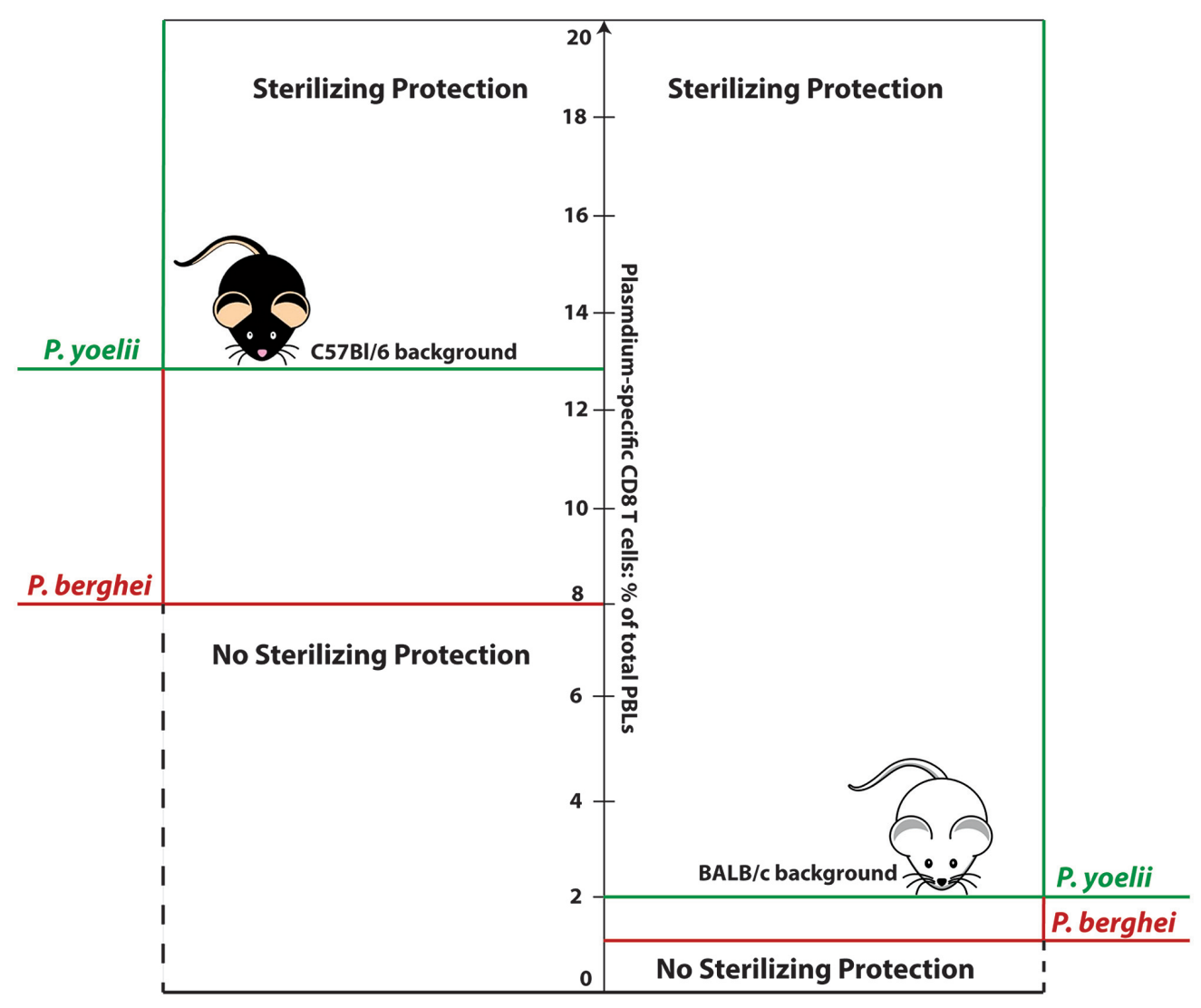

FIGURE 1 | Numerical thresholds required for memory CD8 T-cell-mediated sterilizing protection from sporozoite challenge: influence of host background genes and Plasmodium strain. Sterilizing protection of BALB/C mice against $P$. berghei or $P$. yoelii requires numbers of antigen- (CS-derived epitope) specific memory
CD8 T-cell equivalent to $\sim 1 \%$ or $2 \%$ of total PBLs, respectively. Sterilizing protection of mice with $\mathrm{C} 57 \mathrm{BI} / 6$ background requires substantially higher CD8 T-cell numbers: $\sim 8 \%$ of total PBLs in the case of $P$. berghei infection, or $\sim 13 \%$ in the case of $P$ yoelii infection.

\section{INFLUENCE OF UNRELATED INFECTIONS ON MAINTENANCE OF Plasmodium-SPECIFIC MEMORY CD8 T CELLS AND PROTECTION}

In the parts of the world where malaria is endemic, infections with various, malaria-unrelated pathogens are also very common (Corbett et al., 2006; Magambo et al., 2006; Hotez and Kamath, 2009). Therefore, it is very likely that the burden of multiple infections has a substantial impact on the maintenance of both numbers and quality of Plasmodium-specific memory CD8 T cells, the major mediators of protection against liver-stage infection (Selin et al., 1996, 1999; Welsh and Selin, 2009). The phenomenon of attrition of antigen-specific memory CD8 T-cell populations has been described earlier in the context of infections with unrelated viruses (Selin et al., 1996, 1999). Specifically, these authors showed that antigen-specific memory CD8 T cells induced in mice by exposure to a viral infection underwent a decrease in frequency and numbers upon subsequent infection with unrelated viruses.

Investigating to which extent memory CD8 T cells are affected by unrelated infections and whether this potential effect can be reversed is of high relevance as it may provide basic information about development and reshaping of Plasmodium-specific immune response and useful guidelines for development of successful vaccine regimens.
In a separate study, we showed that exposure of mice to infections with unrelated pathogens (e.g., LCMV, LM, VacV, MHV-1) following DC-LM CS prime/boost vaccination induces dramatic decreases in frequency, but also in total numbers of antigen-specific memory CD8 T cells (Schmidt and Harty, 2011). Furthermore, more pronounced overall attrition was observed in the subpopulation of effector memory (Tem) compared to central memory $(\mathrm{Tcm}) \mathrm{T}$ cells. This change in subset composition may have substantial functional consequences, as we previously suggested that Tem, but not Tcm are closely correlated with protection against sporozoite challenge (Schmidt et al., 2010). These drastic changes in numbers and composition of antigen-specific memory CD8 T-cell subsets resulted in seriously compromised protection, as only $10 \%$ of immunized mice exposed to malaria-unrelated infections were protected against sporozoite challenge. Importantly, the induced attrition did not hamper the capacity of memory CD8 T cells to expand upon antigen re-encounter. In line with this, a single-booster immunization after virus-induced attrition induced expansion of the memory population and restored the subpopulation structure (Tem vs. $\mathrm{Tcm}$ ), and, most importantly, the protection against sporozoite challenge. 
As multiple pathogens are endemic to the same geographic areas as malaria, it is very likely that vaccination-induced memory CD8 T-cell response will undergo a certain degree of attrition, which poses an additional concern for successful vaccination. The finding that despite the attrition, memory CD8 T cells remain responsive to booster vaccination, suggests that the use of prime and regular booster vaccination approach may be critical to preserve long-term immunity against malaria.

\section{INDUCTION OF CD8 T CELLS BY WHOLE-PARASITE VACCINES BROADENING OF THE ANTIGEN REPERTOIRE BY VACCINATION WITH RAS: IMPACT ON NUMERICAL THRESHOLDS}

To date, only two immunization approaches have demonstrated a capacity to induce sterile protection against liver-stage malaria in both humans and animal models. Recently, an immunization approach based on multiple, low-dose inoculations of wt sporozoites through mosquito bites in individuals were also continually treated with the antimalarial drug chloroquine drug was evaluated. This approach was shown to successfully protect humans from a subsequent mosquito bit challenge (Roestenberg et al., 2011). The second approach is RAS vaccination, which still represents the gold standard of antimalarial protection (Clyde et al., 1973; Edelman et al., 1993; Hoffman et al., 2002). Nevertheless, critical parameters of protection, such as quantity and quality of the RAS-induced memory CD8 T-cell remain unknown.

After establishing that sterile immunity against sporozoite challenge in mice vaccinated with a single Plasmodium CS epitope requires mobilization of a large portion of total memory CD8+ T-cell pool (Schmidt et al., 2008), we decided to probe the hypothesis that broadening the antigenic targets would decrease the threshold of memory CD8 T cells required for protection. Multiple, low-magnitude, immune responses against broad range of antigenic determinants may have a superior protective capacity in comparison to monospecific response. For this purpose, the RAS vaccination regime was applied (Schmidt et al., 2010). To overcome the lack of well-defined MHC class I-restricted antigenic determinants, we took advantage of a surrogate activation marker approach recently described by our laboratory for measuring CD8 T-cell responses to bacterial or viral pathogens (Rai etal., 2009). Activation of effector or memory antigenspecific CD8 $\mathrm{T}$ cells by exposure to viral or bacterial infection induces down regulation of $\mathrm{CD} 8 \alpha$ and up-regulation of CD11a. Importantly, these changes are stable in well-defined, antigenspecific CD8 T cells for the life of the laboratory mouse. This approach allowed us for the first time to follow and quantitatively and qualitatively characterize RAS-specific memory CD8 T-cell response and to assess the induced protection in multiple mouse strains.

To our surprise, broadening of the CD8 T-cell antigenic repertoire by vaccination with RAS did not measurably decrease the numerical threshold of memory cells required for protection (Schmidt et al., 2010). As shown by specific antibody depletion studies, the protection depended on CD8 T cells but not CD4 T cells (Schmidt et al., 2010). Similarly to our results with CD8 $\mathrm{T}$ cells specific for CS-derived epitopes, sterile protection was highly dependent on the mouse strain and Plasmodium species (Figure 1). Thus, BALB/c mice were relatively "easy" to protect, as a single dose $(20,000)$ of RAS was sufficient to induce sterile protection against $P$. berghei challenge in $>80 \%$ of animals, and a single-booster immunization ensured protection against highly virulent $P$. yoelii challenge. On the other hand, protection observed in $\mathrm{C} 57 \mathrm{Bl} / 6$ mice after a single RAS immunization was absent, despite similar memory CD8 T-cell numbers induced upon vaccination in both mouse strains. Solid, sterile protection of these mice against $P$. berghei sporozoite challenge required multiple booster doses and multiple-fold increase in the magnitude of the CD8 T-cell response. Antigen-specific CD8 T-cell populations representing even $40 \%$ of total CD8 T cells in $\mathrm{B} 6$ mice were not sufficient to protect against $P$. yoelii challenge at a memory time point.

Importantly, application of surrogate activation markers allowed us for the first time to follow and characterize RASspecific memory CD8 T-cell responses and subsequent protection in populations of outbred mice, which may be a better model for genetically diverse human populations. In striking contrast to more or less uniform memory CD8 T-cell responses induced in inbred mice, responses measured in outbred groups varied substantially in magnitude and kinetics among individual animals. Despite wide distribution of immune responses, $>80 \%$ of primeboosted outbred mice were sterilely protected against homologous challenge with $P$. berghei and $P$. yoelii sporozoite infection. Importantly, this finding could potentially predict the distribution of immune responses upon whole-parasite immunization at the level of heterogeneous human populations.

\section{MULTIPLE BENEFITS FROM TARGETING LATE-LIVER-STAGE ANTIGENS}

Recently, a new sporozoite attenuation strategy has been developed, based on a direct manipulation (deletion) of target parasite genes (Mueller et al., 2005b). Such a targeted attenuation method may be superior in comparison to irradiation. While irradiation induces random lesions in DNA molecules and consequently gives rise to genetically diverse population of RAS, genetic manipulation allows for controlled production of well-defined and genetically similar parasites (genetically attenuated parasites - GAP; Chattopadhyay et al., 2009; Kappe et al., 2010). Additionally, while attenuation by irradiation arrests the parasite development at an early liver stage (Menard et al., 2013), targeting specific genes crucial for various metabolic processes allows for more or less custom design of GAP and production of both early- and late-liver-stagearresting parasites (Mueller et al., 2005a,b; Aly et al., 2008). GAP have been tested in multiple pre-clinical studies and induced CD8 T-cell-mediated protection in murine malaria models (Labaied et al., 2007; Tarun et al., 2007).

Given the different degrees of intrahepatic development between early-liver-stage-arrested RAS and GAP and late-liverstage-arrested GAP, we hypothesized that the latter expresses a broader spectrum of antigen determinants, and possibly induces superior protection. Consistent with this notion, vaccination with late-liver-stage-arrested GAP induced higher memory CD8 $\mathrm{T}$-cell responses, which closely correlated with better protection induced in BALB/c, but also C57Bl/6 and Swiss Webster mice (Butler et al., 2011). Importantly, the antigenic specificity of the memory CD8 T-cell immune responses induced by early- and late-liver-stage-arresting vaccines was only partially overlapping. 
From our study, it was clear that late-liver-stage-arresting GAP induce responses against broader spectrum of antigens compared to both RAS and early-liver-stage-arrested GAP. Thus, we concluded that superior protection induced upon vaccination with late-liver-stage-arrested GAP was based on the broadening of the antigenic repertoire.

These findings are particularly important in the light of recent finding of Tarun et al. (2008), who predicted that late-liver-stage and blood-stage parasites have substantial overlap in their antigen pools. This would mean that vaccination with late-liver-stagearrested GAP may also induce cross-stage protection. Indeed, mice vaccinated with high numbers of late-liver-stage-arrested GAP, but not RAS, displayed high degree of protection against both liverand blood-stage challenge (Butler et al., 2011).

Targeting a broad spectrum of antigens, particularly the ones shared by different developmental stages of the parasite, may increase the protective efficacy of a vaccine (Table 1). Not only do late-liver-stage-arrested GAP represent a promising vaccine candidate, but these parasites may also serve as an important model for determining novel CD8 T-cell antigens, which might be exploited for design of new generation of cross-protective subunit vaccines.

\section{OUTCOME OF RECENT HUMAN TRIALS: NUMBERS DO MATTER}

Although a substantial body of evidence has been collected pointing to the relevance of CD8 T-cell response in controlling liver-stage malaria, current vaccines have displayed rather modest capacity of inducing such responses in humans. The most advanced malaria vaccine is RTS,S, a subunit vaccine based on CS protein, which induces partial protection that correlates with antibody and CD4 T-cell immune responses, but no detectable CD8 T-cell response (Moorthy and Ballou, 2009). In the most recent field clinical trial in children this vaccine displayed $\sim 30 \%$ efficacy against clinical malaria in the target population of 6-12-month-old infants (Agnandji et al., 2012). Thus, there is a need for development of new vaccine candidates with the capacity to elicit CD8 T-cell response, and which can be used alone or in combination with RTS,S.

One of the promising CD8 T-cell-inducing approaches is based on heterologous prime-boost strategies using different viral vectors expressing the same CD8 T-cell-target antigens (Hill et al., 2010). Unfortunately, most of the vaccination regimens that were successful in mice failed when tested in humans (Ockenhouse et al., 1998; Moorthy et al., 2004; Bejon et al., 2006, 2007). Recently, a new promising prime-boost regimen based on chimpanzee adenovirus/MVA expressing Plasmodium thrombospondin adhesive protein (TRAP) fused to a multiple epitopes derived from several malaria antigens has been successfully tested in pre-clinical studies (Colloca et al., 2012), followed by two clinical trials in humans (O'Hara et al., 2012; Ewer et al., 2013). Results of the Phase IIa clinical trial revealed that $20 \%$ of vaccinated subjects had sterilizing immunity against standardized sporozoite infection, and $35 \%$ of vaccines displayed significant delay to a patency, which represent an additional measure of vaccine efficacy (Ewer et al., 2013). Although the degree of protection was modest, a very important finding of the study was the close correlation between the observed protection and the increased percentage of total CD8 T cells producing vaccine antigen-specific IFN- $\gamma$, over that induced in previous vaccine trials. Moreover, the efficiency of protection (sterile protection vs. delay to a patency vs. lack of protection) was dependent on the magnitude of IFN- $\gamma$-producing CD8 T cells, suggesting the existence of protective threshold, which is in line with our finding in a mouse model. The existence of protective CD8 T-cell thresholds may explain the failure of previous vaccination regimens tested in humans (Schmidt et al., 2008, 2010). On the other hand, it remains possible that protective memory CD8 T-cell thresholds in humans may be lower $(<0.3 \%$ of total CD8 T cells) than those we observed in mice ( $>15 \%$ of total CD8 T cells). One of the possible explanations is the prolonged intra-hepatic phase of sporozoite infection in humans (6-8 days), compared to mice (2 days), providing human CD8 T cells with substantially more time for detection and elimination of infected cells, which might decrease the cell numbers required for successful protection.

Another recent human trial confirmed the importance of magnitude of CD8 T-cell response in protection against liverstage malaria. Although it has been known for four decades that vaccination of human subjects with RAS via mosquito bites induces sterile protection against subsequent sporozoite challenge, only recently the first aseptic, radiation-attenuated, metabolically active, purified, cryopreserved, sporozoite vaccine was tested in a clinical trial, when it was injected into human subjects via two standard vaccination routes: subcutaneous (s.c.) and intradermal (i.d.; Epstein etal., 2011). Interestingly, the result of this first RAS clinical trial was the absence of protection in all the subjects, regardless of the dose received. With the hypothesis that the failure in protection was due to the suboptimal vaccination route rather than to lack of vaccine immunogenicity, Seder et al. (2013) performed another clinical trial using the

Table 1 | Comparison between early- and late-liver stage arresting whole-sporozoite vaccines.

\begin{tabular}{|c|c|c|c|c|c|}
\hline Vaccine & $\begin{array}{l}\text { Mode of } \\
\text { inactivation }\end{array}$ & Stage of arrest & $\begin{array}{l}\text { Percentage of } \\
\text { sterilizing protection } \\
\text { after a single dose }\end{array}$ & $\begin{array}{l}\text { Protection against } \\
\text { blood-stage } \\
\text { challenge }\end{array}$ & $\begin{array}{l}\text { Cross-species } \\
\text { protection }\end{array}$ \\
\hline RAS & Irradiation & Early liver & 5 & No & Partial \\
\hline $\operatorname{sap}^{-}$GAP & Mutagenesis & Early liver & 20 & No & - \\
\hline$f a b b^{-}$GAP & Mutagenesis & Late liver & 40 & Yes & Yes \\
\hline
\end{tabular}


intravenous (i.v.) route for the vaccine delivery. The hypothesis was initially tested and confirmed in both non-human primate and a mouse model, as which revealed that i.v. immunization with RAS induces superior protection against sporozoite challenge when compared to s.c. immunization (Epstein et al., 2011). Using the previously described surrogate activation markers to enumerate antigen-specific CD8 T cells, authors showed that i.v. immunization induces immune response of substantially higher magnitude in liver and spleen than s.c. immunization. In line with these findings, and in striking contrast to s.c. vaccination, i.v. vaccination of human subjects induced sterilizing protection in $80 \%$ of subjects receiving the highest vaccine dose. Moreover, the protection correlated with numbers of CD8 T cells producing IFN- $\gamma$ in a majority of protected subjects. These findings demonstrate superior protection induced by i.v. administration of RAS, and suggest induction of sufficient numbers of antigenspecific CD8 $\mathrm{T}$ cells as underlying mechanism of the observed protection.

Together these two studies suggest the translational value of our main finding in a mouse malaria model: the importance of memory CD8 T-cell numbers in sterilizing protection against liver-stage malaria and the potential existence of a definable threshold for sterilizing immunity. Besides being fundamental findings of high importance for further improvement of current and development of new malaria vaccine candidates and vaccination regimens, these findings also justify the use of a mouse as a suitable system for studying basic principles of immunity against pre-erythrocytic malaria stage.

\section{CONCLUDING REMARKS}

In summary, recent information generated in the mouse model of Plasmodium infection sets the cornerstone for further research of CD8 T-cell-mediated protection against liver-stage malaria. The most striking finding is that induction of extremely high numbers of memory CD8 $\mathrm{T}$ cells is a prerequisite for solid, sterile protection. Therefore, developing vaccines or vaccination regimens that will ensure induction of high-magnitude memory CD8 T-cell response against selected, highly protective target antigens is one means for successful immunization. However, it is still not known whether induction of additional immune components, such as CD4 T cells and antibodies, would positively influence the protection, for example, by decreasing the required numbers of CD8 $\mathrm{T}$ cells. Additionally, induction of CD8 $\mathrm{T}$ cells specific for antigens shared by (late) liver-stage and blood-stage parasite is a promising approach toward cross-stage, and possibly cross-species protection. Thus, one focus for future research should be discovery and characterization of such "shared" epitopes. Finally, the precise qualitative features leading to optimal memory CD8 T-cell protection against liver-stage Plasmodium infection remain to be determined.

\section{ACKNOWLEDGMENTS}

The authors thank all the present and former members of Harty laboratory and all of our collaborators for their contributions to the work described herein.

\section{REFERENCES}

Agnandji, S. T., Lell, B., Fernandes, J. F., Abossolo, B. P., Methogo, B. G., Kabwende, A. L., etal. (2012). A phase 3 trial of RTS,S/AS01 malaria vaccine in African infants. N. Engl. J. Med. 367, 2284-2295. doi: 10.1056/NEJMoa12 08394

Aly, A. S., Mikolajczak, S. A., Rivera, H. S., Camargo, N., Jacobs-Lorena, V., Labaied, M., et al. (2008). Targeted deletion of SAP1 abolishes the expression of infectivity factors necessary for successful malaria parasite liver infection. Mol. Microbiol. 69, 152-163. doi: 10.1111/j.1365-2958.2008.06271.x

Badovinac, V. P., Messingham, K. A., Jabbari, A., Haring, J. S., and Harty, J. T. (2005). Accelerated CD8+ T-cell memory and prime-boost response after dendritic-cell vaccination. Nat. Med. 11, 748-756. doi: 10.1038/nm1257

Baer, K., Klotz, C., Kappe, S. H., Schnieder, T., and Frevert, U. (2007). Release of hepatic Plasmodium yoelii merozoites into the pulmonary microvasculature. PLoS Pathog. 3:e171. doi: 10.1371/journal.ppat.0030171

Bejon, P., Mwacharo, J., Kai, O., Mwangi, T., Milligan, P., Todryk, S., et al. (2006). A phase $2 \mathrm{~b}$ randomised trial of the candidate malaria vaccines FP9 ME-TRAP and MVA ME-TRAP among children in Kenya. PLoS Clin. Trials 1:e29. doi: 10.1371/journal.pctr.0010029

Bejon, P., Ogada, E., Mwangi, T., Milligan, P., Lang, T., Fegan, G., et al. (2007). Extended follow-up following a phase $2 \mathrm{~b}$ randomized trial of the candidate malaria vaccines FP9 ME-TRAP and MVA ME-TRAP among children in Kenya. PLoS ONE 2:e707. doi: 10.1371/journal.pone.0000707

Butler, N. S., Schmidt, N. W., and Harty, J. T. (2010). Differential effector pathways regulate memory CD8 $\mathrm{T}$ cell immunity against Plasmodium berghei versus P. yoelii sporozoites. J. Immunol. 184, 2528-2538. doi: 10.4049/jimmunol.09 03529

Butler, N. S., Schmidt, N. W., Vaughan, A. M., Aly, A. S., Kappe, S. H., and Harty, J. T. (2011). Superior antimalarial immunity after vaccination with late liver stage-arresting genetically attenuated parasites. Cell Host Microbe 9, 451-462. doi: 10.1016/j.chom.2011.05.008

Chattopadhyay, R., Conteh, S., Li, M., James, E. R., Epstein, J. E., and Hoffman, S. L. (2009). The effects of radiation on the safety and protective efficacy of an attenuated Plasmodium yoelii sporozoite malaria vaccine. Vaccine 27, 3675-3680. doi: 10.1016/j.vaccine.2008.11.073

Clyde, D. F., Most, H., Mccarthy, V. C., and Vanderberg, J. P. (1973). Immunization of man against sporozite-induced falciparum malaria. Am. J. Med. Sci. 266, 169-177. doi: 10.1097/00000441-197309000-00002

Colloca, S., Barnes, E., Folgori, A., Ammendola, V., Capone, S., Cirillo, A., et al. (2012). Vaccine vectors derived from a large collection of simian adenoviruses induce potent cellular immunity across multiple species. Sci. Transl. Med. 4, 115ra112. doi: 10.1126/scitranslmed.3002925

Corbett, E. L., Marston, B., Churchyard, G. J., and De Cock, K. M. (2006). Tuberculosis in sub-Saharan Africa: opportunities, challenges, and change in the era of antiretroviral treatment. Lancet 367, 926-937. doi: 10.1016/S01406736(06)68383-9

Diosi, P. (2002). Genetic control of innate immune responses against cytomegalovirus: MCMV meets its match. Genes Immun. 3, 501. doi: 10.1038/sj.gene.6363938

Doolan, D. L., and Hoffman, S. L. (1999). IL-12 and NK cells are required for antigen-specific adaptive immunity against malaria initiated by CD8 $+\mathrm{T}$ cells in the Plasmodium yoelii model. J. Immunol. 163, 884-892.

Doolan, D. L., and Hoffman, S. L. (2000). The complexity of protective immunity against liver-stage malaria. J. Immunol. 165, 1453-1462. doi: 10.4049/jimmunol.165.3.1453

Dunachie, S. J., Walther, M., Epstein, J. E., Keating, S., Berthoud, T., Andrews, L., et al. (2006). A DNA prime-modified vaccinia virus ankara boost vaccine encoding thrombospondin-related adhesion protein but not circumsporozoite protein partially protects healthy malaria-naive adults against Plasmodium falciparum sporozoite challenge. Infect. Immun. 74, 5933-5942. doi: 10.1128/IAI.00 590-06

Edelman, R., Hoffman, S. L., Davis, J. R., Beier, M., Sztein, M. B., Losonsky, G., et al. (1993). Long-term persistence of sterile immunity in a volunteer immunized with X-irradiated Plasmodium falciparum sporozoites. J. Infect. Dis. 168, 1066-1070. doi: 10.1093/infdis/168.4.1066

Epstein, J. E., Tewari, K., Lyke, K. E., Sim, B. K., Billingsley, P. F., Laurens, M. B., et al. (2011). Live attenuated malaria vaccine designed to protect through hepatic CD8(+) T cell immunity. Science 334, 475-480. doi: 10.1126/science.1211548 
Ewer, K. J., O’hara, G. A., Duncan, C. J., Collins, K. A., Sheehy, S. H., Reyes-Sandoval, A., et al. (2013). Protective CD8+ T-cell immunity to human malaria induced by chimpanzee adenovirus-MVA immunisation. Nat. Commun. 4, 2836. doi: $10.1038 /$ ncomms 3836

Ferreira, A., Schofield, L., Enea, V., Schellekens, H., Van Der Meide, P., Collins, W. E., et al. (1986). Inhibition of development of exoerythrocytic forms of malaria parasites by gamma-interferon. Science 232, 881-884. doi 10.1126/science. 3085218

Hill, A. V., Reyes-Sandoval, A., O’Hara, G., Ewer, K., Lawrie, A., Goodman, A., et al. (2010). Prime-boost vectored malaria vaccines: progress and prospects. Hum. Vaccin. 6, 78-83. doi: 10.4161/hv.6.1.10116

Hoffman, S. L., Goh, L. M., Luke, T. C., Schneider, I., Le, T. P., Doolan, D. L., et al. (2002). Protection of humans against malaria by immunization with radiationattenuated Plasmodium falciparum sporozoites. J. Infect. Dis. 185, 1155-1164. doi: $10.1086 / 339409$

Hotez, P. J., and Kamath, A. (2009). Neglected tropical diseases in sub-saharan Africa: review of their prevalence, distribution, and disease burden. PLoS Negl Trop. Dis. 3:e412. doi: 10.1371/journal.pntd.0000412

Hsieh, C. S., Macatonia, S. E., O'garra, A., and Murphy, K. M. (1995). T cell genetic background determines default $\mathrm{T}$ helper phenotype development in vitro. J. Exp. Med. 181, 713-721. doi: 10.1084/jem.181.2.713

Ishikawa, T., Yamada, H., Oyamada, A., Goshima, F., Nishiyama, Y., and Yoshikai, Y. (2009). Protective role of Fas-FasL signaling in lethal infection with herpes simplex virus type 2 in mice. J. Virol. 83, 11777-11783. doi: 10.1128/JVI.01006-09

Jobe, O., Lumsden, J., Mueller, A. K., Williams, J., Silva-Rivera, H., Kappe, S. H. et al. (2007). Genetically attenuated Plasmodium berghei liver stages induce sterile protracted protection that is mediated by major histocompatibility complex Class I-dependent interferon-gamma-producing CD8+ T cells. J. Infect. Dis. 196, 599_ 607. doi: 10.1086/519743

Kappe, S. H., Vaughan, A. M., Boddey, J. A., and Cowman, A. F. (2010). That was then but this is now: malaria research in the time of an eradication agenda. Science 328, 862-866. doi: 10.1126/science. 1184785

Labaied, M., Harupa, A., Dumpit, R. F., Coppens, I., Mikolajczak, S. A., and Kappe, S. H. (2007). Plasmodium yoelii sporozoites with simultaneous deletion of P52 and P36 are completely attenuated and confer sterile immunity against infection. Infect. Immun. 75, 3758-3768. doi: 10.1128/IAI.00225-07

Lanar, D. E., Tine, J. A., De Taisne, C., Seguin, M. C., Cox, W. I., Winslow, J. P., et al. (1996). Attenuated vaccinia virus-circumsporozoite protein recombinants confer protection against rodent malaria. Infect. Immun. 64, 1666-1671.

Magambo, J., Njoroge, E., and Zeyhle, E. (2006). Epidemiology and control of echinococcosis in sub-Saharan Africa. Parasitol. Int. 55(Suppl.) S193-S195. doi: 10.1016/j.parint.2005.11.029

Meis, J. F., Verhave, J. P., Jap, P. H., Sinden, R. E., and Meuwissen, J. H. (1983). Malaria parasites - discovery of the early liver form. Nature 302, 424-426. doi $10.1038 / 302424 \mathrm{a} 0$

Menard, R., Tavares, J., Cockburn, I., Markus, M., Zavala, F., and Amino, R. (2013) Looking under the skin: the first steps in malarial infection and immunity. Nat. Rev. Microbiol. 11, 701-712. doi: 10.1038/nrmicro3111

Miller, L. H., Ackerman, H. C., Su, X. Z., and Wellems, T. E. (2013). Malaria biology and disease pathogenesis: insights for new treatments. Nat. Med. 19, 156-167. doi: $10.1038 / \mathrm{nm} .3073$

Moorthy, V. S., and Ballou, W. R. (2009). Immunological mechanisms underlying protection mediated by RTS,S: a review of the available data. Malar. J. 8, 312. doi: 10.1186/1475-2875-8-312

Moorthy, V. S., Imoukhuede, E. B., Milligan, P., Bojang, K., Keating, S., Kaye, P., et al. (2004). A randomised, double-blind, controlled vaccine efficacy trial of DNA/MVA ME-TRAP against malaria infection in Gambian adults. PLoS Med. 1:e33. doi: 10.1371/journal.pmed.0010033

Mueller, A. K., Camargo, N., Kaiser, K., Andorfer, C., Frevert, U., Matuschewski, K., et al. (2005a). Plasmodium liver stage developmental arrest by depletion of a protein at the parasite-host interface. Proc. Natl. Acad. Sci. U.S.A. 102, 3022-3027. doi: 10.1073/pnas.0408442102

Mueller, A. K., Labaied, M., Kappe, S. H., and Matuschewski, K. (2005b). Genetically modified Plasmodium parasites as a protective experimental malaria vaccine. Nature 433, 164-167. doi: 10.1038/nature03188

Nussenzweig, R. S., Vanderberg, J., Most, H., and Orton, C. (1967). Protective immunity produced by the injection of X-irradiated sporozoites of Plasmodium berghei. Nature 216, 160-162. doi: 10.1038/216160a0
Nussenzweig, R. S., Vanderberg, J. P., Most, H., and Orton, C. (1969). Specificity of protective immunity produced by $\mathrm{x}$-irradiated Plasmodium berghei sporozoites. Nature 222, 488-489. doi: 10.1038/222488a0

Ockenhouse, C. F., Sun, P. F., Lanar, D. E., Wellde, B. T., Hall, B. T., Kester, K., et al. (1998). Phase I/IIa safety, immunogenicity, and efficacy trial of NYVAC-Pf7, a pox-vectored, multiantigen, multistage vaccine candidate for Plasmodium falciparum malaria. J. Infect. Dis. 177, 1664-1673. doi: 10.1086/ 515331

O’Hara, G. A., Duncan, C. J., Ewer, K. J., Collins, K. A., Elias, S. C., Halstead, F. D., et al. (2012). Clinical assessment of a recombinant simian adenovirus ChAd63: a potent new vaccine vector. J. Infect. Dis. 205, 772-781. doi: 10.1093/infdis/ jir850

Potocnjak, P., Yoshida, N., Nussenzweig, R. S., and Nussenzweig, V. (1980). Monovalent fragments $(\mathrm{Fab})$ of monoclonal antibodies to a sporozoite surface antigen (Pb44) protect mice against malarial infection. J. Exp. Med. 151, 1504-1513. doi: 10.1084/jem.151.6.1504

Raeder, R. H., Barker-Merrill, L., Lester, T., Boyle, M. D., and Metzger, D. W. (2000). A pivotal role for interferon-gamma in protection against group A streptococcal skin infection. J. Infect. Dis. 181, 639-645. doi: 10.1086/315281

Rai, D., Pham, N. L., Harty, J. T., and Badovinac, V. P. (2009). Tracking the total CD8 $\mathrm{T}$ cell response to infection reveals substantial discordance in magnitude and kinetics between inbred and outbred hosts. J. Immunol. 183, 7672-7681. doi: 10.4049/jimmunol.0902874

Renggli, J., Hahne, M., Matile, H., Betschart, B., Tschopp, J., and Corradin, G. (1997). Elimination of $P$. berghei liver stages is independent of Fas (CD95/ApoI) or perforin-mediated cytotoxicity. Parasite Immunol. 19, 145-148. doi: 10.1046/j.1365-3024.1997.d01-190.x

Rodrigues, E. G., Claassen, J., Lee, S., Wilson, J. M., Nussenzweig, R. S., and Tsuji, M. (2000). Interferon-gamma-independent CD8 + T cell-mediated protective antimalaria immunity elicited by recombinant adenovirus. Parasite Immunol. 22, 157-160. doi: 10.1046/j.1365-3024.2000.00289.x

Rodrigues, E. G., Zavala, F., Eichinger, D., Wilson, J. M., and Tsuji, M. (1997). Single immunizing dose of recombinant adenovirus efficiently induces CD8+ $\mathrm{T}$ cell-mediated protective immunity against malaria. J. Immunol. 158, 12681274.

Rodrigues, M. M., Cordey, A. S., Arreaza, G., Corradin, G., Romero, P., Maryanski, J. L., et al. (1991). CD8+ cytolytic T cell clones derived against the Plasmodium yoelii circumsporozoite protein protect against malaria. Int. Immunol. 3, 579-585. doi: 10.1093/intimm/3.6.579

Roestenberg, M., Teirlinck, A. C., Mccall, M. B. B., Teelen, K., Makamdop, K. N., Wiersma, J., et al. (2011). Long-term protection against malaria after experimental sporozoite inoculation: an open-label follow-up study. Lancet 377, 1770-1776. doi: 10.1016/S0140-6736(11)60360-7

Sadoff, J. C., Ballou, W. R., Baron, L. S., Majarian, W. R., Brey, R. N., Hockmeyer, W. T., et al. (1988). Oral Salmonella typhimurium vaccine expressing circumsporozoite protein protects against malaria. Science 240, 336-338. doi: $10.1126 /$ science. 3281260

Schmidt, N. W., Butler, N. S., Badovinac, V. P., and Harty, J. T. (2010). Extreme CD8 $\mathrm{T}$ cell requirements for anti-malarial liver-stage immunity following immunization with radiation attenuated sporozoites. PLoS Pathog. 6:e1000998. doi: 10.1371/journal.ppat.1000998

Schmidt, N. W., Butler, N. S., and Harty, J. T. (2009). CD8 T cell immunity to Plasmodium permits generation of protective antibodies after repeated sporozoite challenge. Vaccine 27, 6103-6106. doi: 10.1016/j.vaccine.2009.08.025

Schmidt, N. W., Butler, N. S., and Harty, J. T. (2011). Plasmodium-host interactions directly influence the threshold of memory CD8 T cells required for protective immunity. J. Immunol. 186, 5873-5884. doi: 10.4049/jimmunol.1100194

Schmidt, N. W., and Harty, J. T. (2011). Cutting edge: attrition of Plasmodiumspecific memory CD8 $\mathrm{T}$ cells results in decreased protection that is rescued by booster immunization. J. Immunol. 186, 3836-3840. doi: 10.4049/jimmunol.1003949

Schmidt, N. W., Podyminogin, R. L., Butler, N. S., Badovinac, V. P., Tucker, B. J., Bahjat, K. S., et al. (2008). Memory CD8 T cell responses exceeding a large but definable threshold provide long-term immunity to malaria. Proc. Natl. Acad. Sci. U.S.A. 105, 14017-14022. doi: 10.1073/pnas.0805452105

Schofield, L., Villaquiran, J., Ferreira, A., Schellekens, H., Nussenzweig, R., and Nussenzweig, V. (1987). Gamma interferon, CD8+ T cells and antibodies required for immunity to malaria sporozoites. Nature 330, 664-666. doi: 10.1038/330664a0 
Sedegah, M., Weiss, W. W., and Hoffman, S. L. (2007). Cross-protection between attenuated Plasmodium berghei and P. yoelii sporozoites. Parasite Immunol. 29, 559-565. doi: 10.1111/j.1365-3024.2007.00976.x

Seder, R. A., Chang, L. J., Enama, M. E., Zephir, K. L., Sarwar, U. N., Gordon, I. J., et al. (2013). Protection against malaria by intravenous immunization with a nonreplicating sporozoite vaccine. Science 341, 1359-1365. doi: 10.1126/science. 1241800

Selin, L. K., Lin, M. Y., Kraemer, K. A., Pardoll, D. M., Schneck, J. P., Varga, S. M., et al. (1999). Attrition of T cell memory: selective loss of LCMV epitope-specific memory CD8 T cells following infections with heterologous viruses. Immunity 11, 733-742. doi: 10.1016/S1074-7613(00)80147-8

Selin, L. K., Vergilis, K., Welsh, R. M., and Nahill, S. R. (1996). Reduction of otherwise remarkably stable virus-specific cytotoxic $\mathrm{T}$ lymphocyte memory by heterologous viral infections. J. Exp. Med. 183, 2489-2499. doi: 10.1084/jem.183. 6.2489

Shrestha, B., Wang, T., Samuel, M. A., Whitby, K., Craft, J., Fikrig, E., et al. (2006). Gamma interferon plays a crucial early antiviral role in protection against West Nile virus infection. J. Virol. 80, 5338-5348. doi: 10.1128/JVI.00 274-06

Shrestha, B., Zhang, B., Purtha, W. E., Klein, R. S., and Diamond, M. S. (2008). Tumor necrosis factor alpha protects against lethal West Nile virus infection by promoting trafficking of mononuclear leukocytes into the central nervous system. J. Virol. 82, 8956-8964. doi: 10.1128/JVI.01118-08

Sturm, A., Amino, R., Van De Sand, C., Regen, T., Retzlaff, S., Rennenberg, A., et al. (2006). Manipulation of host hepatocytes by the malaria parasite for delivery into liver sinusoids. Science 313, 1287-1290. doi: 10.1126/science. 1129720

Sturm, A., and Heussler, V. (2007). Live and let die: manipulation of host hepatocytes by exoerythrocytic Plasmodium parasites. Med. Microbiol. Immunol. 196, 127 133. doi: 10.1007/s00430-007-0044-3

Tarun, A. S., Dumpit, R. F., Camargo, N., Labaied, M., Liu, P., Takagi, A., et al. (2007). Protracted sterile protection with Plasmodium yoelii pre-erythrocytic genetically attenuated parasite malaria vaccines is independent of significant liver-stage persistence and is mediated by CD8+ T cells. J. Infect. Dis. 196, 608-616. doi: $10.1086 / 519742$

Tarun, A. S., Peng, X., Dumpit, R. F., Ogata, Y., Silva-Rivera, H., Camargo, N., et al. (2008). A combined transcriptome and proteome survey of malaria parasite liver stages. Proc. Natl. Acad. Sci. U.S.A. 105, 305-310. doi: 10.1073/pnas.07107 80104

Todryk, S. M., and Hill, A. V. (2007). Malaria vaccines: the stage we are at. Nat. Rev. Microbiol. 5, 487-489. doi: 10.1038/nrmicro1712
Trapani, J. A., and Smyth, M. J. (2002). Functional significance of the perforin/granzyme cell death pathway. Nat. Rev. Immunol. 2, 735-747. doi: $10.1038 /$ nri911

Tsuji, M., Miyahira, Y., Nussenzweig, R. S., Aguet, M., Reichel, M., and Zavala, F. (1995). Development of antimalaria immunity in mice lacking IFN-gamma receptor. J. Immunol. 154, 5338-5344.

Weiss, W. R., Good, M. F., Hollingdale, M. R., Miller, L. H., and Berzofsky, J. A. (1989). Genetic control of immunity to Plasmodium yoelii sporozoites. J. Immunol. 143, 4263-4266.

Weiss, W. R., Sedegah, M., Beaudoin, R. L., Miller, L. H., and Good, M. F. (1988). $\mathrm{CD} 8+\mathrm{T}$ cells (cytotoxic/suppressors) are required for protection in mice immunized with malaria sporozoites. Proc. Natl. Acad. Sci. U.S.A. 85, 573-576. doi 10.1073/pnas.85.2.573

Weiss, W. R., Sedegah, M., Berzofsky, J. A., and Hoffman, S. L. (1993). The role of CD4+ T cells in immunity to malaria sporozoites. J. Immunol. 151, 26902698.

Welsh, R. M., and Selin, L. K. (2009). Attrition of memory CD8 T cells. Nature 459, E3-E4; discussion E4. doi: 10.1038/nature08091

WHO. (2011). World Malaria Report 2011. Geneva: World Health Organization, Available at: http://www.who.int/malaria/world_malaria_report_2011

Yoshida, N., Nussenzweig, R. S., Potocnjak, P., Nussenzweig, V., and Aikawa, M. (1980). Hybridoma produces protective antibodies directed against the sporozoite stage of malaria parasite. Science 207, 71-73. doi: 10.1126/science.6985745

Conflict of Interest Statement: The authors declare that the research was conducted in the absence of any commercial or financial relationships that could be construed as a potential conflict of interest.

Received: 28 March 2014; accepted: 19 May 2014; published online: 06 June 2014. Citation: Van Braeckel-Budimir N and Harty JT (2014) CD8 T-cell-mediated protection against liver-stage malaria: lessons from a mouse model. Front. Microbiol. 5:272. doi: 10.3389/fmicb.2014.00272

This article was submitted to Microbial Immunology, a section of the journal Frontiers in Microbiology.

Copyright (C) 2014 Van Braeckel-Budimir and Harty. This is an open-access article distributed under the terms of the Creative Commons Attribution License (CC BY). The use, distribution or reproduction in other forums is permitted, provided the original author(s) or licensor are credited and that the original publication in this journal is cited, in accordance with accepted academic practice. No use, distribution or reproduction is permitted which does not comply with these terms. 